\title{
Dependent Variables in the Privacy-Related Field: A Descriptive Literature Review
}

\author{
Jakob Wirth \\ University of Bamberg, Germany \\ jakob.wirth@uni-bamberg.de
}

\begin{abstract}
As privacy is an ongoing issue of both society and research, there is a tremendous amount of research on privacy in the domain of information systems. A plethora of these studies has been conducted on privacy-related dependent variables. This descriptive literature review summarizes used dependent variables and gives a detailed analysis of the variables including the research setting, used theories, used methodologies, and used research designs. Results show among others that 1) some dependent variables are under-researched, 2) the majority is using intention to disclose as their dependent variable, 3) many articles are not grounded in a basic underlying theory and 4) the majority is using cross-sectional surveys as their research design. Based on the results several recommendations for future research are given, including to use certain dependent variables, to focus on actual disclosure behaviour and to conduct longitudinal studies.
\end{abstract}

\section{Introduction}

"If this is the age of information then privacy is the issue of our times" [1]. Indeed, there are several examples, which prove this citation and indicate that the privacy of individuals in the field of information systems (IS) is threatened such as data breaches [2], governmental agencies spying on citizens [3] or cyber attacks [4]. This also leads to disadvantages such as discrimination or manipulation [1]. To better understand how individuals react to privacy-related topics in light of such privacy threats, research in the domain of IS focuses on different privacy-related issues. Thereby, research tries to explain dependent variables which represent variables that are affected by other, independent variables to explain a certain issue [5]. Examples of dependent variables are disclosure of information, risk or trust [6].
Having an overview on what dependent variables have been used would lead to an overview on what previous studies have tried to explain in the privacyrelated field. Such a latest state of the art on used dependent variables would also provide other academics a starting point when doing research on privacy in the domain of IS. Furthermore, it might identify research gaps [7], e.g. dependent variables which have not been used in particular research settings. Then recommendations for future research could be given to push research in the privacy-related IS-area forward and thusly to have a better overall understanding of what has been tried to explain in the privacy-related field.

To gain such overviews of the tremendous amount of research on privacy, several literature reviews have already been conducted $[6,8-10]$. They have provided different overviews, e.g. the antecedents-privacy concerns-outcomes (APCO) model [6], a general overview of the central role of privacy concerns [9] or used theories in the privacy domain [11]. However, a clear overview on the latest state of the art of used dependent variables in the domain of IS is missing. The conducted literature reviews might also be outdated since privacy is an ongoing field which changes over time [3, 12]. Hence, additional research might have been conducted since the last literature reviews which has not been aggregated in prior literature reviews. We will thusly try to fill these research gaps by asking the following research question:

What is the latest state of the art on dependent variables in the privacy-related field in the domain of information systems?

To answer the research question, we conducted a descriptive literature review [7] in the area of IS and examined 142 articles. Among others, our results reveal that previous studies have used a plethora of different dependent variables. Implications for research include to also use under-represented dependent variables, to more research on actual disclosure behaviour and to conduct more longitudinal studies. 
The study proceeds with a short theoretical background about privacy-related research in section two followed by the methodology of our study in section three to better understand our results which are presented in section four. The results are discussed in section five by presenting implications.

\section{Privacy-related research}

Privacy is defined as the interest an individual has in controlling or at least having an influence on controlling her disclosed information [8].

To measure privacy, and to find out how privacy has an influence on other concepts, privacy-related research in the domain of IS has tested different dependent variables. A dependent variable is defined as the outcome concept, which is determined by independent variables [5]. In a research study, a dependent variable can also be an antecedent of another dependent variable but is then called a mediator. However, in our study, we treat dependent variables as all the variables, which do only serve as dependent variables but not as mediators.

To explain dependent variables, privacy-related research has among others used diverse theories. One of the theories is the privacy calculus [13] which explains disclosure of information as a dependent variable. Disclosure of information is an important dependent variable as without disclosing information individuals' privacy is usually not threatened [14]. The theory implies that individuals disclose information if the benefits of disclosure outweigh the costs of disclosure.

For a better differentiation of disclosure of information, the dependent variable should be separated into actual disclosure behaviour and intention to disclose [6]. Usually, the intention is a good predictor of behaviour [15], however, in privacy research as well as in other research areas [16], an intention-behaviour gap has been recognized [17]. This implies that individuals intend to behave privacy conform but then actually behave contrary.

Besides the intention-behaviour gap, there is the privacy paradox. This states that individuals reveal a huge amount of information despite being concerned about their privacy $[18,19]$. Privacy concerns thereby refer to the level of worry about the threat to the privacy of an individual [20]. The privacy paradox can also be partly explained by situational factors [21] which might occur through the research setting. For example, the situation of individuals is different when researching on social networking sites (SNS) than when researching on an IS in a healthcare environment due to the sensitivity of the information disclosed [22].
The named theories and variables play a major role in privacy research and help the reader to better understand the answer to our research question. To answer our research question, we conducted a literature review.

\section{Methodology}

The goal of this research study is to research on used dependent variables to identify research gaps. As recommended by previous literature we therefore conducted a descriptive literature review. A descriptive literature review aims to uncover the latest state of the art of a particular instantiation in a particular area. It is therefore not comprehensive but rather focuses on a particular area. We focus on the latest state of the art of used dependent variables in the privacy-related field in the domain of IS [7].

\subsection{Scope and conduction of the literature review}

During the entire process of our literature review, we kept with previous guidelines [7, 23]. As suggested by them, one should start the literature review with the analysis of a set of journals. As our literature review is done in the area of IS, we started our literature search in the AIS basket of eight [24]. Moreover, we included ICIS Proceedings and ECIS Proceedings as two major conferences in the IS community [25]. We did not limit our review to a specific period of time. The search was done by searching in the title, abstract, and keywords. We took "privacy" as our search term because when this term is neither used in the title nor in the abstract or keywords, the article will probably not deal with privacy-related research [9].

\subsection{Selection procedure}

In the first run, we identified 308 articles. In a first selection, we then read the title, abstract and keywords and dismissed all articles, which do not deal with privacy in their study. For example, some articles are using privacy as an example but focus on a completely different concept. Further, 219 articles remained after the first selection. Then, in a second selection we thoroughly read all articles and dismissed all articles, when at least one of the following points applied: 1) accounting for the organizational but not individual perspective, 2) editorials, 3) panels, 4) commentaries, 5) teaching cases, 6) research in progress, 7) literature reviews, 8) sole conceptual papers, 9) scale developments, 10) dealing with technical or mathematical operations to understand privacy, 11) call for papers or 12) articles which have been 
published on a conference but a very similar article has then been published in a journal. After the second selection, 84 articles remained. We then performed a forward- and backward-search to identify additional articles which are not published in the top journals or conferences. The backward-search was done by checking all references of every article. Scanning and selection was done as explained above. Forward search was also done for all 84 articles by using Web of Science. Again, the same procedure applied. By performing backward- and forward-search, 58 additional articles were identified for our literature review. Hence, this literature review deals with 142 articles which serve as the basis to answer our research question (see Table 1).

Table 1. Research scope and number of articles identified

\begin{tabular}{|c|c|c|c|}
\hline $\begin{array}{l}\text { Journal/ } \\
\text { conference }\end{array}$ & $\begin{array}{|lr|}\# & \text { of } \\
\text { articles } & \text { in } \\
\text { the } & 1 \text { st } \\
\text { run } & \\
\end{array}$ & $\begin{array}{l}\# \text { of articles } \\
\text { after 1st } \\
\text { selection }\end{array}$ & \begin{tabular}{|l}
$\#$ of \\
articles \\
after 2nd \\
selection \\
\end{tabular} \\
\hline EJIS & 11 & 9 & 6 \\
\hline JAIS & 10 & 8 & 4 \\
\hline MISQ & 23 & 18 & 6 \\
\hline ISR & 18 & 16 & 8 \\
\hline ISJ & 8 & 6 & 2 \\
\hline JIT & 2 & 2 & 1 \\
\hline JSIS & 11 & 9 & 3 \\
\hline JMIS & 16 & 9 & 3 \\
\hline ICIS Proceedings & 123 & 97 & 34 \\
\hline ECIS Proceedings & 86 & 45 & 17 \\
\hline & $\sum 308$ & $\sum 219$ & $\sum 84$ \\
\hline \multicolumn{3}{|c|}{ Backward- and forward-search: } & $\sum 58$ \\
\hline \multicolumn{3}{|c|}{$\begin{array}{l}\# \text { of articles, which are used for the literature } \\
\text { review }\end{array}$} & $\sum \underline{142}$ \\
\hline
\end{tabular}

\subsection{Coding procedure}

As this is a descriptive literature review, we analyzed the results by collecting, codifying and analyzing the frequency of topics, methods or theories to produce quantitative results [7]. In particular, as exhibited in section four, the coding procedure was done within the following topics: the demographics and the research setting, the used dependent variables, used theories, and the used methodology including the research design.

First, the research setting was examined by checking on the methodology section. If the study is explicitly stating the research setting, such as a SNS setting, it was coded accordingly. In cases, where the research setting was not explicitly stated we categorized it as follows: All studies which are conducted using a social media technology such as Facebook, Twitter or YouTube are coded as SNS.
Location based research settings are all settings where location data was examined, for example, data from a GPS module in a smartphone. A healthcare research setting was coded when the study was done in the context of hospitals, medical data or similar. Purchasing as a research setting was used when the research was conducted in a commercial setting, such as examining information about credit card transfers or e-commerce in general. For reasons of parsimony, all other research settings, for example, general websites, were put under the category other. Studies can also be done in more than one research setting, for example, studies can research on Facebook in the context of hospitals. The research setting of such a study would be coded as SNS and healthcare.

Second, the used dependent variables were examined. Equal dependent variables with different labels were combined into a single dependent variable. For example, the dependent variable privacy concerns has been measured by using terms such as privacy concerns, and individual privacy concerns which were both coded as privacy concerns [6].

Third, used theories were examined by asking in how far the study is based on one or more basic theories. If the study is not grounded on a basic theory, then the study was coded as not applicable (N/A).

Fourth, the used methodology was examined. This was done by investigating if the study conducted a survey, an experiment, a vignette/scenario-based study, a qualitative study, a conjoint analysis or an observation in the field. If studies have conducted more than one methodology they were coded accordingly. In addition, we asked for the research design, i.e. if the study was conducted via a crosssectional or a longitudinal study. A cross-sectional study is conducted when data was gathered once versus a longitudinal study where data was gathered over at least two points of time [26].

Fifth, actual disclosure behaviour was analysed in more depth. All studies, which reported on objective data measurements and studies which asked for past self-reported disclosure on SNS were coded as actual disclosure behaviour because such past self-reported data on SNS does not significantly differ from actual disclosure [27]. Furthermore, antecedents of actual disclosure behaviour were analysed.

The coding process was done from one academic researcher who has experience in the privacy-related field. To avoid organisational blindness and to crosscheck results, a second researcher again coded the results. Then both researchers discussed the results where the coding process revealed diverse results. Based on the discussion a final coding was done over all articles. Due to space restrictions, the final coded concept matrix including information going beyond 
the mentioned figures is available online ${ }^{1}$ or upon request.

\section{Results}

The results of the literature review are presented in the following sections by firstly providing an overview of demographics and the research setting. Then, dependent variables, used theories, used methodologies and the research design are exhibited.

\subsection{Demographics and research setting}

Figure 1 provides a graphical representation of the year of publication. There is an increase of number of articles from the year 2004, rising a peak in the years 2013 and 2015. Furthermore, there are more conference proceedings in recent years than in the years before where journal articles are dominating.

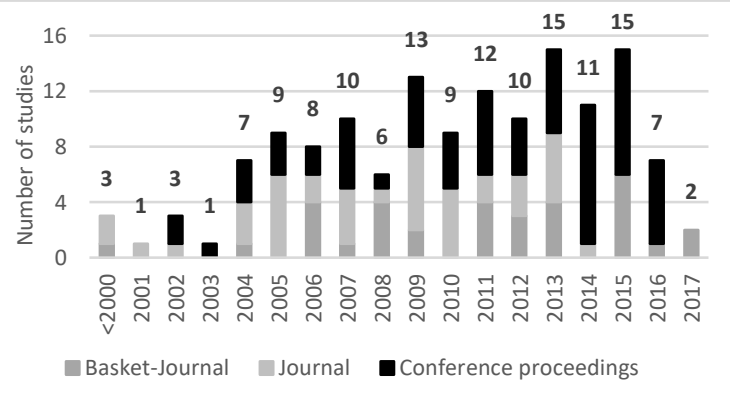

Figure 1 Demographics

Figure 2 reveals details about the used research settings. Studies were categorized into a SNS setting, a purchase setting, a location based setting, and a healthcare setting. About one-half of the studies were conducted in other research settings.

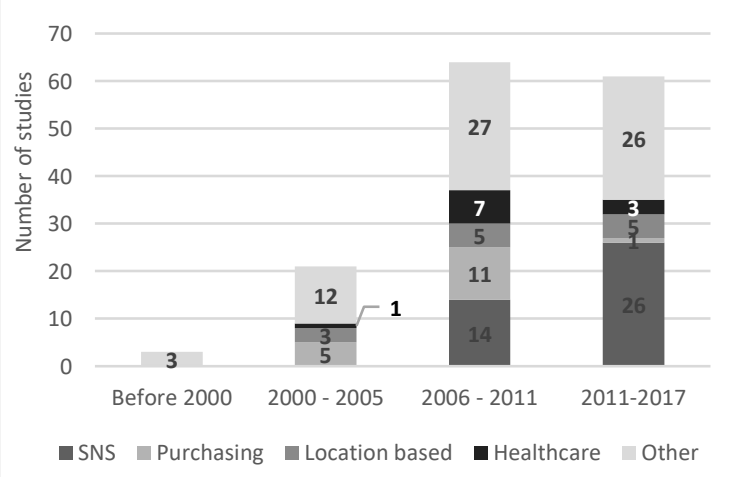

Figure 2 Research setting

The results show that there have been no studies on SNS until 2005. Then, from 2006 on there is a steady

\footnotetext{
${ }^{1}$ https://isdl.uni-bamberg.de/online-appendix/cm hicss.pdf
}

increase in studies researching on SNS. Other research settings thereby were cut back over time, for instance, healthcare or purchasing. Location based settings remain on a constant level. What these research studies have been researching on is well indicated by the used dependent variables.

\subsection{Dependent variables}

Figure 3 gives an overview of used dependent variables in previous privacy-related research in the domain of IS. The dependent variables are categorized in either behaviour-related or psychological-related dependent variables. The former refers to reactions of individuals in terms of their behaviour, e.g. disclosure of information. The latter is about psychological reactions of individuals [28], e.g. privacy concerns.

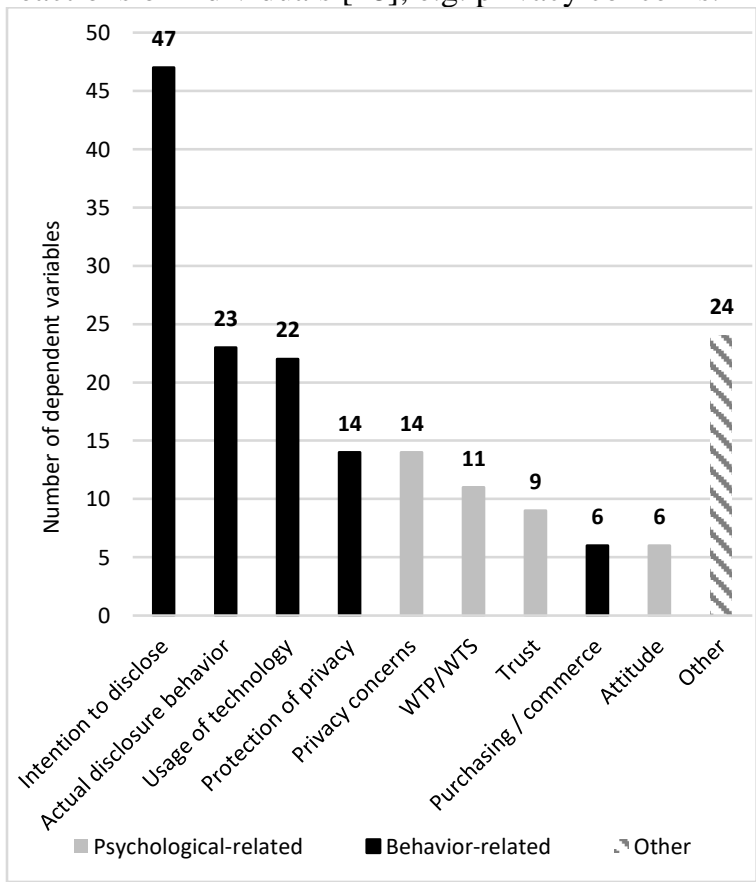

Figure 3 Dependent variables used by prior research

As one can see, behaviour-related dependent variables represent the majority of dependent variables. Intention to disclose is the most used variable, followed by actual disclosure behaviour which includes observations of actual behaviour as well as self-reported past behaviour in SNS settings. Usage of technology and protection of privacy have been used third or fourth most, respectively. Usage of technology includes variables such as the usage of cloud software. Protection of privacy refers to 
variables such as taking private action for the protection of ones' privacy.

Then, psychological-related dependent variables follow, including privacy concerns and willingness to pay (WTP) for privacy or willingness to sell (WTS) ones' privacy. WTP represents a maximal monetary value an individual is willing to pay to protect her privacy whereas WTS presents a minimal monetary value, an individual demands to sell her privacy. Furthermore, trust as an additional psychologicalrelated dependent variable is presented.

Purchasing as a behaviour-related dependent variable follows ahead of attitude of an individual as a psychological-related dependent variable. In addition, 24 other variables which could not be categorized into one of the named categories were identified.

All in all, this part reveals that there are several dependent variables used by previous privacy research. In addition, it is shown that the majority is using behaviour-related dependent variables, with intention to disclose and actual disclosure behaviour being the most used ones. However, also psychological-related dependent variables have been used, especially privacy concerns and WTP/WTS which represent the two most used ones of psychological-related dependent variables. As these four dependent variables represent the two most used dependent variables in either a behaviour-related category or a psychological-related category, a detailed analysis of these four variables is conducted in the following section.

\subsection{Used theories}

In this section, used theories of previous privacy research are presented. As shown in Figure 4, several basic theories have been used by previous research. The privacy calculus is the most used theory. The following theories have been used at last three times to explain a dependent variable: The social exchange theory [29] which describes how an individual feels about a relationship; the protection motivation theory [30], which is about the process how an individual protects herself; the communication privacy management (CPM) theory [14] is about the way why individuals reveal or hide information and the elaboration likelihood model [31], which is about the state of an individual in respect to a specific subject she receives information about. 43 times other theories have been used less than three times each to explain dependent variables. For 76 dependent variables, no basic theory has been used.

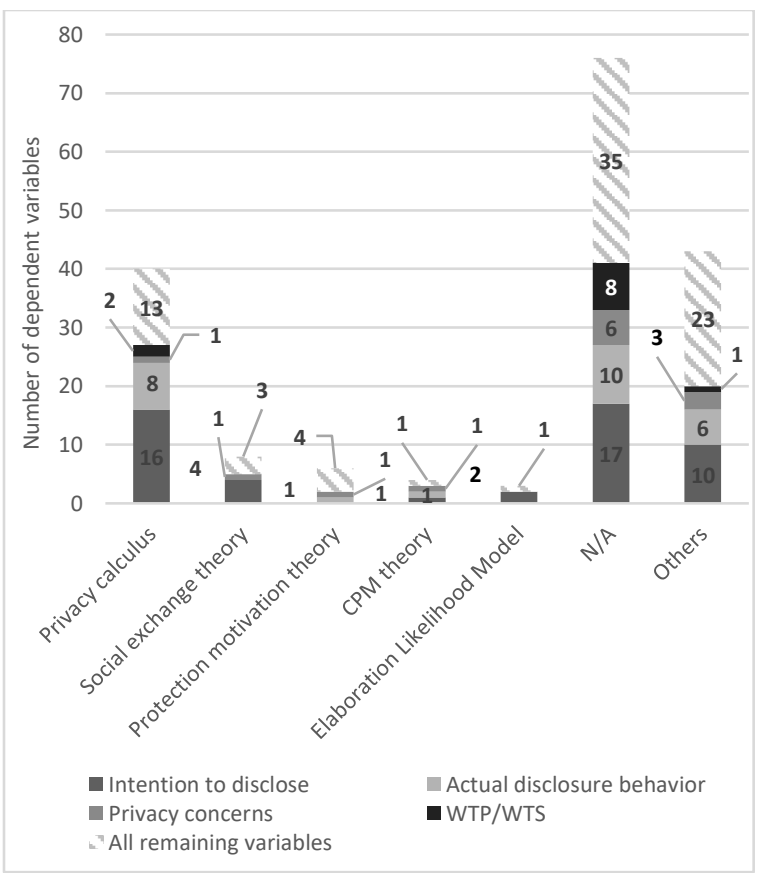

Figure 4 Used theories in relation to dependent variables

Setting the most used variables of behaviour- and psychological-related dependent variables (see Figure 3 ) in relationship to the used theories, then Figure 4 displays that the privacy calculus has mainly been used to explain intention to disclose and actual disclosure behaviour. Three times it is used to either explain privacy concerns or WTP/WTS. Social exchange theory, protection motivation theory, CPM theory and the elaboration likelihood model have been used less often. Also, not every theory has been used to explain every single dependent variable. For example, none of the four theories has been used to explain WTP/WTS. Also, the elaboration likelihood model is not used to explain actual disclosure behaviour or intention to disclose.

To better understand how the analysed research studies conducted their studies we also examined the methodology of the studies and the used research design.

\subsection{Used methodology and research design}

In this section, the conducted methodology and the used research design are presented. Figure 5 depicts the used methodologies of the analysed studies as well as their research design. As one can see, surveys are used by most of the studies (111), followed by experiments (57), vignette/scenario-based analyses (22), qualitative study designs (12), conjoint analyses (2) and observings in the field (1). 
Besides the used methodology, we also analysed the research design of each study i.e. was the study conducted by a cross-sectional study or by a longitudinal study. The results reveal that the overwhelming majority is using cross-sectional studies (191 times) in contrast to longitudinal studies (13 times). In addition, Figure 5 reveals that in eight out of thirteen longitudinal studies, surveys were the used methodology, whereas experiments were used three times and a qualitative methodology was used two times.

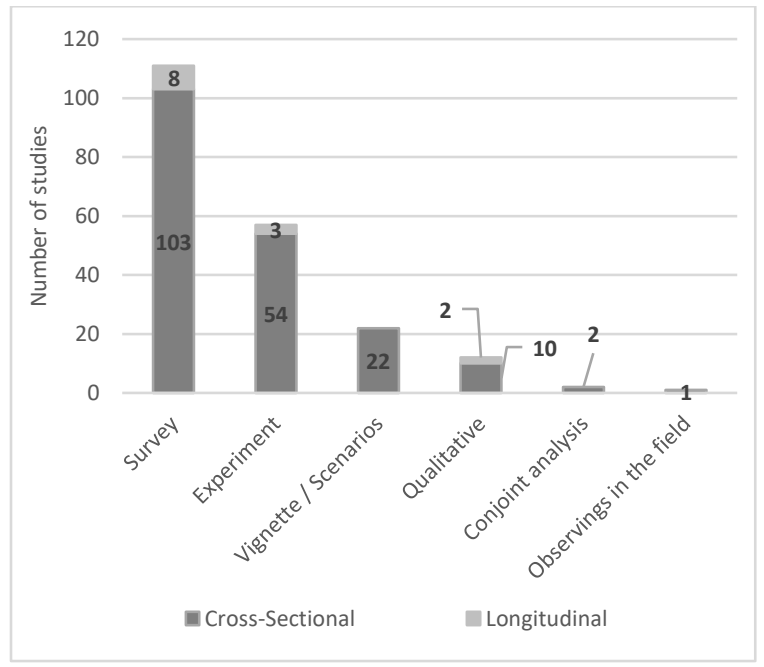

Figure 5 Methodology and research design

All in all, the aforementioned sections reveal the demographics (Figure 1), the research setting in relation to the year of publication (Figure 2), used dependent variables (Figure 3), used theories in relation to the dependent variables (Figure 4) as well as used methodologies and the research design of each study (Figure 5).

The results have thereby identified actual disclosure behaviour as the second most used dependent variable in previous privacy research. As there have been several calls in previous privacy research for more investigation on that variable $[6,8]$ and as there is the privacy paradox which is directly related to actual disclosure behaviour but not fully explained, yet [21], we have a deeper look at that variable in the following section.

\subsection{Further analysis of actual disclosure behaviour}

Actual disclosure behaviour is an important variable which is also shown by several calls for more research on that variable in the year 2011 [6, 8]. Actual disclosure behavior refers to the measurements of objective data measures or self-reported past disclosure behavior on SNS [27].

Therefore, we investigated the year of publication for that variable to research on in how far these calls have been answered. 14 out of 24 studies on actual disclosure behaviour have been conducted after the year 2011.

To receive a better understanding of actual disclosure behaviour we also investigated the antecedents. Table 2 provides an overview in how far antecedents do have a positive, a non-significant or a negative influence on actual disclosure behavior. Other variables are displayed in the concept matrix.

Table 2. Influence of antecedents on actual disclosure behavior

\begin{tabular}{|l|l|l|l|}
\hline Antecedent & Positive & $\begin{array}{l}\text { Non- } \\
\text { significant }\end{array}$ & Negative \\
\hline $\begin{array}{l}\text { Privacy } \\
\text { concerns }\end{array}$ & n/a & 3 & 4 \\
\hline Benefits & 4 & 1 & n/a \\
\hline Trust & 1 & 2 & n/a \\
\hline
\end{tabular}

As shown in Table 2 privacy concerns have a negative influence on actual disclosure behavior in four studies, whereas three studies did not find a significant influence. Four studies show a positive influence of benefits whereas one study reveals a nonsignificant influence. One study also shows that trust positively influences actual disclosure behavior whereas two studies did not find a significant influence.

The studies which have shown a significant influence of privacy concerns on actual disclosure behaviour counteract with the privacy paradox [17]. Therefore, we analysed these results in even more detail and revealed that two of them studied on SNS, and the other two of them on domains which were not categorized by this study. The studies were carried out by conducting cross-sectional studies and using surveys or experiments.

To understand how the aforementioned results have implications for theory in the domain of IS, we discuss the results in the following section.

\section{Discussion}

The results reveal that the number of published privacy-related articles in the domain of IS has increased over the last 15 years and now remains on a rather constant level. One should also consider that several new publications are to be expected in recent years as articles from these years have not been cited that much, yet, and therefore might not have been identified during the backward-search. 
The rising number of articles indicates that the importance of privacy in the IS related field has emerged. This trend might even hold on since privacy has become an important asset and topic in society [1, $3,12]$. However, although much research has already been done on privacy in the domain of IS, there are still several recommendations, future research might grab to push the understanding of privacy in the IS related field forward.

First, more research on under-represented dependent variables. The results have shown that some dependent variables, such as intention to disclose or privacy concerns, have often been researched on. Although we do not say that future research on these dependent variables is unnecessary, we want to pinpoint to dependent variables where less research has been done such as purchasing behavior or WTP/WTS. For example, research on purchasing behavior is important because previous research has shown that privacy plays an important role in the context of purchasing online [32]. In addition, there is also scarcity on other dependent variables which are not displayed in Figure 3. For example, previous research has pointed out that mass surveillance by governmental agencies is an important topic in todays' society and massively threatens the privacy of individuals [33]. However, our literature review does hardly find studies dealing with that topic. Research could therefore for example ask why individuals accept or reject mass surveillance [34].

Second, no overemphasis on social networking sites as a research setting. The results (Figure 2) have revealed that previous studies have been done in different research settings, such as social networking sites (SNS), location-based settings or healthcare. It was shown that especially the SNS research setting was used many times from the time frame of 2007 on which is also the time in which the number of privacyrelated articles began to leap. This might be due to Facebook which is the biggest SNS [35] and which was released in the year 2004. Thereby, although the number of articles has risen, other research settings have slightly been neglected. Although we do agree on that SNS is an important research area, scholars should not create their general privacy understanding solely based on SNS. We rather argue to not neglect SNS but also to focus on other research areas e.g. healthcare. Especially in that research setting highly sensitive information is transferred [22] and privacy might be one of the big obstacles in a prospective society [36]. Consequently, academics might want to put research in that or also other research areas for a better and more holistic privacy-related understanding.

Third, more consideration of the intentionbehaviour gap. The results (Figure 3 ) have revealed that the majority of studies in the domain of IS has used intention to disclose as their dependent variable. Usually, intention is a good predictor of behaviour [15], however, there is an intention-behaviour gap which shows that intention to disclose does not always adequately predict actual disclosure behaviour [17]. This might also be due to a general attitude-behaviour gap [37]. The importance of actual disclosure behaviour is even more emphasized when considering that after recent calls for more research on actual disclosure behaviour [6,8] several articles researching on that dependent variable have been published since then. Future studies in the area of IS should follow that lead and try to use actual disclosure behaviour as their dependent variable if possible, instead of intention to disclose.

In addition, Figure 3 reveals that behaviour-related dependent variables are at the tops in the area of IS research. However, also several psychological-related dependent variables have been used, e.g. privacy concerns. Psychological-related dependent variables are important for a better understanding of individuals' perceptions. However, even more interesting is in how far these psychological-related dependent variables have an influence on the actual behaviour of individuals. Therefore, we encourage researchers in the domain of IS to take one step further and investigate in how far psychological-related dependent variables actually influence behaviour-related dependent variables.

Fourth, more usage of a basic theory. The results have revealed that about one half of the analysed studies in the area of IS do not use a basic underlying theory (see Figure 4). However, usually empirical studies have the aim to contribute to existing theories and to support hypotheses. Therefore, having a basic underlying theory significantly contributes to the quality of a paper. This can be done by showing what we know until today, i.e. using a current theory, and then to show how one extends that theory by the results of the study. Therefore, the aim is not to just use a basic underlying theory but the aim is to use such a basic underlying theory to better develop new or extended theories [38]. It is thusly somewhat surprising that only one half of the conducted studies is doing so. We do not want researchers to not enter new paths for novel insights into the issue of privacy in the domain of IS. However, using a basic theory does not prohibit researchers from doing so but helps to put their results on a firm grounding [39].

The results have additionally revealed that the privacy calculus [13] is the most used theory [11]. Although the privacy calculus has clearly put the understanding of privacy in the IS field forward [11] and should therefore not be neglected, researchers 
might also take new perspectives by relying on theories other than the privacy calculus. Our results have revealed diverse theories, which have been used by previous research. Hence, these theories might be used as a starting point for researchers in the field of IS to put the privacy-related understanding forward. For example, using a privacy-related theory such as the CPM theory [14] might reveal additional insights because it could explain why individuals disclose information which is also a request made by previous research [8].

But also other non-privacy theories, such as the social exchange theory [29] could help in a better understanding of privacy-related research in the domain of IS. For example, social exchange theory as a non-privacy theory can bring up new perspectives and ideas the privacy research needs to explain undeclared occurrences such as the privacy paradox [21]. Social exchange theory might then be used to better understand why an individual is exchanging information with some individuals and with some others not, depending on the relationship to those individuals. Also, other theories, which are depicted in the concept matrix might gain new insights to better understand dependent variables and other occurrences in the privacy-related field of IS research.

Fifth, more attention on longitudinal studies and less used methodologies. Our results reveal that most of the studies in the IS domain use a crosssectional research design in contrast to a longitudinal research design (see Figure 5). A longitudinal study thereby refers to a study where there is research on the same set of individuals more than once whereas a cross-sectional study is about a study of individuals which took place for only one time [26]. This is especially a problem because also the majority of the studies has used surveys in their methodology. When conducting surveys, usually researchers build a theoretical research model, hypothesize causal relationships between variables, and then try to find support for these hypotheses through a survey. However, these causal relationships cannot be proven with a cross-sectional study where only correlations are identified [40]. This is because causal relationships need a temporal order, i.e. A needs to be before B to imply a causal order between A and B. Since there is no temporal order in a cross-sectional study which is conducted at the same point of time, causal relationships cannot be identified. However, longitudinal studies can help in inferring causal relationships because they are conducted at different points of time [41]. We therefore encourage researchers in the domain of IS to conduct more longitudinal studies to better identify causal relationships between antecedents and the dependent variable, especially when conducting surveys.

Besides doing longitudinal studies, researchers in the IS domain can also think about using a different methodology other than just surveys. Experiments or vignette/scenario based analyses [42] have already been used by several researchers but more research using one of these two methodologies might gain additional insights into dependent variables. This is because when using one of these two methodologies, the researcher can then better control for the actual antecedents of dependent variables and isolate other variables which might also have an influence on the dependent variable. Through this, the actual antecedents of the dependent variable can better be identified. Also, the usage of qualitative methodologies would give researchers the possibility to dig into fields which are more under-researched such as the privacy paradox or the intention-behaviour gap [43].

Sixth, more research to better understand the privacy paradox. As explained in the theoretical background, the privacy paradox states that privacy concerns do not have an influence on actual disclosure behaviour of individuals [17]. However, as revealed by our results (Table 2), there are several studies in the domain of IS proving a significant influence of privacy concerns on actual disclosure behaviour. Although we do not claim that Table 2 provides a full meta-analytic review about antecedents of actual disclosure behavior, our results still reveal that the privacy paradox does not always exist. As shown in the results section, those studies, which prove an influence of privacy concerns on actual disclosure behaviour used different research settings and methodologies. Hence, other reasons which explain the privacy paradox, e.g. moderating effects [8], might be used by researchers in the area of IS to better being able to explain the privacy paradox [21]. Recent research has also called for a more fine-granular split of actual disclosure behaviour, for example referring to psychological, informational and social behaviour [44]. Doing so might also help in a better understanding of the privacy paradox.

Overall, this study reveals several implications for researchers in the privacy-related field in the IS domain to put the understanding of privacy forward. Although the study has some limitations, which are explained in the following section, we do not think that these limitations mainly infer the mentioned implications. 


\section{Limitations, future research and conclusion}

One limitation of this study refers to the problem of subjectivity. Hence, different keywords could have been used, different categorizations might have been built and some might have categorized articles differently than we did. However, as the results were cross-checked with another researcher, we do not think that others would come up with complete different results. Another limitation refers to the fact that the study was solely done in the context of IS. Hence, knowledge of other areas such as psychology or economy have rather been neglected in this study. However, the results have only been generalized to the field of IS-research. Still, additional insights might be gained when extending the literature review on fields other than IS-research. As this was not the scope of the review, we also did not diversify the concept of disclosure of information by different aspects such as falsification [20]. An additional study might gain deeper insights into that concept. However, we still think that our results are valid at this point as such different concepts all relate to disclosure of information.

Overall, the goal of our literature review was to give an overview on used dependent variables in privacy research in the domain of IS. Based on our results we provide some recommendations, other researchers might follow: 1) Doing more research on under-researched dependent variables such as purchasing behavior in an online context or acceptance/rejection of mass surveillance 2) not overemphasizing SNS as the only research setting, 3) considering the intention-behaviour gap when researching on disclosure of information and also considering doing research on the influence of psychological variables on behavioral outcomes, 4) putting more emphasis on a basic underlying theory, 5) using longitudinal studies and 6) doing more research on the privacy paradox.

Besides these recommendations, future research can use these results as a starting point to e.g. better identify how to measure privacy, which is still a nebulous endeavor [6]. One could also use this research to create a detailed analysis what has changed in the privacy research domain within the last years especially after recent detailed review articles [6]. We also used self-reported past disclosure behavior on SNS as actual disclosure behavior as both do not mainly differ from each other [27]. To gain more insights into actual disclosure behavior, scholars might conduct more research to find out in how far this also applies to contexts other than SNS and also in how far actual disclosure behavior is and should be measured.
Furthermore, future research could perform a detailed analysis of all mentioned studies conducting experiments to find out in how far these experiments actually depict real world scenarios, e.g., by accounting for actual risks or benefits of disclosure. Moreover, future research could also use a basic privacy theory such as the CPM theory [14] to identify further research gaps. Another opportunity is to include used antecedents of the dependent variables and undertake a meta-analysis of the relationships of the study to gain insights into the influence of antecedents on the dependent variables.

\section{References}

1. Acquisti, A., Brandimarte, L., Loewenstein, G.: Privacy and human behavior in the age of information. Science 347, 509-514 (2015)

2. Victor, D.: Security breach at toy maker vtech includes data on children, http://www.nytimes.com/2015/12/01/business/security -breach-at-toy-maker-vtech-includes-data-onchildren.html?_r=0

3. Gidda, M.: Edward Snowden and the NSA files timeline,

http://www.theguardian.com/world/2013/jun/23/edwar d-snowden-nsa-files-timeline

4. Abbott, C.: Cyber-Attacks: A Problem In 2016, Still A Problem in 2017, http://www.natlawreview.com/article/cyber-attacksproblem-2016-still-problem-2017

5. Fraenkel, J.R., Wallen, N.E.: How to design and evaluate research in education. McGraw-Hill, New York (1993)

6. Smith, J.H., Dinev, T., Xu, H.: Information privacy research: An interdisciplinary review. MIS Quarterly 35, 980-1015 (2011)

7. Paré, G., Trudel, M.-C., Jaana, M., Kitsiou, S.: Synthesizing information systems knowledge A typology of literature reviews. Information \& Management 52, 183-199 (2015)

8. Bélanger, F., Crossler, R.E.: Privacy in the digital age: A review of information privacy research in information systems. MIS Quarterly 35, 1017-1042 (2011)

9. Li, Y.: Empirical Studies on Online Information Privacy Concerns: Literature Review and an Integrative Framework. Communications of the Association for Information Systems 28, 453-496 (2011)

10. Pavlou, P.A.: State of the information privacy literature: Where are we now and where should we go? MIS Quarterly 35, 977-988 (2011)

11. Li, Y.: Theories in online information privacy research. A critical review and an integrated framework. Decision Support Systems 54, 471-481 (2012)

12. Sledge, M.: The Snowden Effect: 8 Things That Happened Only Because Of The NSA Leaks, http://www.huffingtonpost.com/2014/06/05/edwardsnowden-nsa-effect_n_5447431.html 
13. Dinev, T., Hart, P.: An extended privacy calculus model for e-commerce transactions. Information Systems Research 17, 61-80 (2006)

14. Petronio, S.: Communication privacy management theory: What do we know about family privacy regulation? Journal of Family Theory \& Review 2, 175 196 (2010)

15. Fishbein, M., Ajzen, I.: Belief, attitude, intention, and behavior. An introduction to theory and research. Addison-Wesley Pub. Co., Reading, Mass (1975)

16. Maier, C., Laumer, S., Eckhardt, A., Weitzel, T.: Using User Personality to explain the Intention-Behavior Gap and Changes in Beliefs: A Longitudinal Analysis. In: Huang, M.-H., Piccoli, G., Sambamurthy, V. (eds.) Proceedings of the 33rd International Conference on Information Systems. Orlando, FL (2012)

17. Norberg, P.A., Horne, D.R., Horne, D.A.: The Privacy Paradox: Personal Information Disclosure Intentions versus Behaviors. Journal of Consumer Affairs 41, 100-126 (2007)

18. Taddicken, M.: The 'Privacy Paradox'in the Social Web: The Impact of Privacy Concerns, Individual Characteristics, and the Perceived Social Relevance on Different Forms of Self-Disclosure. Journal of Computer-Mediated Communication 19, 248-273 (2014)

19. Barnes, S.B.: A privacy paradox: Social networking in the United States. First Monday 11 (2006)

20. Son, J.-Y., Kim, S.S.: Internet Users' Information Privacy-Protective Responses: A Taxonomy and a Nomological Model. MIS Quarterly 32, 503-529 (2008)

21. Kokolakis, S.: Privacy attitudes and privacy behaviour: A review of current research on the privacy paradox phenomenon. Computers \& Security, 122-134 (2015)

22. Anderson, C.L., Agarwal, R.: The Digitization of Healthcare: Boundary Risks, Emotion, and Consumer Willingness to Disclose Personal Health Information. Information Systems Research 22, 469-490 (2011)

23. Webster, J., Watson, R.T.: Analyzing the past to prepare for the future: Writing a literature review. Management Information Systems Quarterly 26, xiii-xxiii (2002)

24. Lowry, P.B., Moody, G.D., Gaskin, J., Galletta, D.F., Humphreyes, S., Barlow, J.B., Wilson, D.: Evaluating Journal Quality and the Association for Information Systems (AIS) Senior Scholars' Journal Basket Via Bibliometric Measures: Do Expert Journal Assessments Add Value? MIS Quarterly 37, 993-1012 (2013)

25. vhb-jourqual: Teilrating Wirtschaftsinformatik, http://vhbonline.org/en/service/jourqual/vhb-jourqual3/teilrating-wi/

26. Kim, S.S., Malhotra, N.K.: A longitudinal model of continued IS use: An integrative view of four mechanisms underlying postadoption phenomena. Management Science 51, 741-755 (2005)

27. Hampton, K., Goulet, L.S., Marlow, C. and Rainie, L.: Why most Facebook users get more than they give, http://www.pewinternet.org/2012/02/03/why-mostfacebook-users-get-more-than-they-give/

28. Tarafdar, M., Tu, Q., Ragu-Nathan, T.S.: Impact of Technostress on End-User Satisfaction and
Performance. Journal of Management Information Systems 27, 303-334 (2010)

29. Emerson, R.M.: Social exchange theory. Annual review of sociology, 335-362 (1976)

30. Rogers, R.W., Prentice-Dunn, S.: Protection motivation theory. In: Handbook of health behavior research 1: Personal and social determinants, pp. 113-132. Plenum Press, New York, NY, US (1997)

31. Petty, R.E., Wegener, D.T.: The elaboration likelihood model: Current status and controversies. In: S. Chaiken Y. Trope (ed.) Dual-process theories in social psychology, pp. 37-72. Guilford Press, New York, NY, US (1999)

32. Dinev, T.: Why would we care about privacy? European Journal of Information Systems 23, 97-102 (2014)

33. Dinev, T., Hart, P., Mullen, M.R.: Internet privacy concerns and beliefs about government surveillance An empirical investigation. The Journal of Strategic Information Systems 17, 214-233 (2008)

34. Reddick, C.G., Chatfield, A.T., Jaramillo, P.A.: Public opinion on National Security Agency surveillance programs. A multi-method approach. Government Information Quarterly 32, 129-141 (2015)

35. Statista.com: Leading social networks worldwide as of April 2016, ranked by number of active users (in millions), http://www.statista.com/statistics/272014/globalsocial-networks-ranked-by-number-of-users/

36. Mukherjee, S.: Why Privacy Is a Big Obstacle In the Digital Health Revolution, http://fortune.com/2016/11/01/fitbit-ceo-privacybrainstorm-health/

37. Fazio, R.H., Roskos-Ewoldsen, D.R.: Acting as We Feel: When and How Attitudes Guide Behavior. In: Green, T.C.B.M.C. (ed.) Persuasion: Psychological insights and perspectives, 2nd ed, pp. 41-62. SAGE Publications, Inc, Thousand Oaks, CA, US (2005)

38. Straub, D.W.: Editor's Comments: Why Top Journals Accept Your Paper. MIS Quarterly 33, iii-x (2009)

39. Gregor, S.: Theory - still king but needing a revolution! J Inf Technol 29, 337-340 (2014)

40. Davis, J.A.: The logic of causal order. Sage (1985)

41. Rindfleisch, A., Malter, A.J., Ganesan, S., Moorman, C.: Cross-Sectional versus Longitudinal Survey Research: Concepts, Findings, and Guidelines. Journal of Marketing Research 45, 261-279 (2008)

42. Shaw, J.C., Wild, E., Colquitt, J.A.: To justify or excuse?: A meta-analytic review of the effects of explanations. Journal of Applied Psychology 88, 444458 (2003)

43. Hofmann, S.: Becoming friends with the government A qualitative analysis of citizens' decision to 'like' government profiles on Facebook. In: Benbasat, I., Andersen, N.B., Sencer, A. (eds.) Proceedings of the 24th European Conference on Information Systems, pp. 1-15. Istanbul, Turkey (2016)

44. Dienlin, T., Trepte, S.: Is the privacy paradox a relic of the past? An in-depth analysis of privacy attitudes and privacy behaviors. European Journal of Social Psychology 45, 285-297 (2015) 